\title{
Intercalated disk remodeling in $\delta$-sarcoglycan-deficient hamsters fed with an $\alpha$-linolenic acid-enriched diet
}

\author{
LAURA MASUELLI $^{1}$, PAOLA TRONO ${ }^{2}$, LAURA MARZOCCHELLA ${ }^{1}$, MARIE-AGNES MROZEK $^{2}$, \\ CAMILLA PALUMBO $^{2}$, MARILENA MINIERI ${ }^{3}$, FELICIA CAROTENUTO ${ }^{3}$, ROBERTA FIACCAVENTO ${ }^{3}$, \\ ALESSANDRA NARDI $^{4}$, FABIO GALVANO $^{5}$, PAOLO DI NARDO $^{3}$, ANDREA MODESTI $^{2}$ and ROBERTO BEI ${ }^{2}$ \\ ${ }^{1}$ Department of Experimental Medicine, University of Rome 'Sapienza'; Departments of ${ }^{2}$ Experimental Medicine and \\ Biochemical Sciences, ${ }^{3}$ Internal Medicine, and ${ }^{4}$ Mathematics, University of Rome 'Tor Vergata', Rome; \\ ${ }^{5}$ Department of Biochemistry, Medical Chemistry and Molecular Biology, University of Catania, Catania, Italy
}

Received August 1, 2007; Accepted September 10, 2007

\begin{abstract}
Cardiomyocyte intercalated disks of $\delta$-sarcoglycandeficient cardiomyopathic hamsters (CMPHs) exhibit a pathological accumulation of the $\mathrm{N}$-cadherin/catenin complex. CMPHs fed with an $\alpha$-linolenic acid (ALA)-enriched diet (CMPH/FS) display an extended longevity compared to those fed with a standard diet (CMPH/PT) owing to, among others, the amelioration of both cardiac tissue structure and myocardial function. The present investigation was aimed at evaluating whether and to what extent the ALA-enriched diet affects the remodeling of CMPH cardiomyocyte intercalated disks and the expression of molecules, including $\mathrm{N}$-cadherin, catenins and connexin 43 (CX43), involved in their organization. Western blot and immunohistochemical analysis demonstrated that the expression of $\mathrm{N}$-cadherin, $\alpha$ - and $\beta$-catenin is significantly reduced in cardiomyocyte intercalated disks of $\mathrm{CMPH} / \mathrm{FS}$ vs. CMPH/PT and is lowered to levels similar to those found in healthy hamsters (GSH/PT), as well as transmission electron microscopy indicated that the cardiomyocyte intercalated disk ultrastructure is also re-established in CMPH/FS. In addition, the delocalization of CX43 as well as the presence of gap junctions were detectable at the lateral plasmamembrane of CMPH/FS cardiomyocytes, while the expression of myocardial CX43 was markedly reduced in both CMPH/PT and CMPH/FS, as compared to GSH/PT. Collectively, the present results demonstrate a substantial effect of an ALA-enriched diet on cardiomyocyte intercalated disk structure and molecular composition and further supports the beneficial effects of $\omega-3$ polyunsatured fatty acids
\end{abstract}

Correspondence to: Professor Roberto Bei, Department of Experimental Medicine and Biochemical Sciences, University 'Tor Vergata', Via Montpellier 1, I-00133 Rome, Italy

E-mail: bei@med.uniroma2.it

Key words: intercalated disks, $\mathrm{N}$-cadherin/catenin, $\alpha$-linolenic acid, cardiomyopathy, diet in the prevention of potentially dangerous arrhythmias in cardiac diseases.

\section{Introduction}

Cardiomyocyte intercalated disks are cell structures containing molecular complexes that play a fundamental role in the anchorage of the myofibrillar apparatus and cytoskeleton to the plasmamembrane as well as in cardiac impulse propagation (1-3). Three different types of specialized junctions are contained within the intercalated disks: adherens junctions, desmosomes and gap junctions (1-4). The adherens junctions connect the plasmamembrane to the actin cytoskeleton and ensure solid cell adhesion (1-4). Components of cardiomyocyte adherens junctions include a) $\mathrm{N}$-cadherin, a transmembrane protein responsible for homophilic cell to cell adhesion, b) $\alpha_{-}, \beta-$ and $\gamma$-catenin, which bind the cytoplasmic tail of $\mathrm{N}$-cadherin and are implicated in signaling, and c) catenin-related proteins, such as vinculin and $\alpha$-actinin $(3,5-8)$. Desmosomes are multiprotein structures composed of cadherins, plakophilins and plakins, that support structural and functional integrity between adjacent cardiomyocytes, participate in signal transduction pathways and may transmit force between cardiomyocytes (9). Gap junctions ensure the electrical coupling and metabolic signal transduction through proteins of the connexin family, among which connexin 43 (CX43) is the main isoform present in the working myocardium $(10,11)$. The mutation or deficiency of the adherens junction components cause cardiomyopathies $(9,12-15)$.

The Syrian $\delta$-sarcoglycan-deficient hamster (UMX7.1 strain) is an extensively used animal model of hypertrophic cardiomyopathy (16-21). Sarcoglycans are members of the sarcolemmal dystrophin-glycoprotein complex (DGC) that links cytoskeleton and extracellular matrix. The integrity of DGC is essential for the viability of muscle cells and defects of its components generate sarcolemmal instability leading to cardiomyocyte damage and necrosis (18). In $\delta$-sarcoglycandeficient hamsters (CMPHs), the cardiomyocyte/basal membrane junction was perturbed and intercalated disks deranged with concomitant substantial accumulation of $\mathrm{N}$ - 
cadherin and $\beta$-catenin (21). In addition, a moderate increase in $\alpha$-catenin, but not in $\gamma$-catenin, was observed (21). In a recent study, most of the $\delta$-sarcoglycan deficiency-related cardiac damage was prevented by feeding CMPHs from weaning to death with an $\alpha$-linolenic acid (ALA)-enriched diet; as a consequence, CMPHs displayed a longer survival as compared to those fed with a standard diet $(293 \pm 144.8$ vs. $175 \pm 56$ days) (20). The present study was, thus, aimed at evaluating whether and to what extent the ALA-enriched diet can affect the remodeling of hamster intercalated disks and the expression of molecules, including $\mathrm{N}$-cadherin, catenins and connexin 43 , involved in their organization.

\section{Materials and methods}

Animal model and dietary treatment. CMPHs (strain UM$\mathrm{X} 7.1$ ), carrying a $\delta$-sarcoglycan (SG) gene deletion, were used and compared with healthy Golden Syrian hamsters (GSHs) bred under the same conditions. Three different groups of hamsters were evaluated: CMPHs and GSHs fed with a standard pellet chow diet (PT) (Rieper, Bolzano, Italy) and CMPH fed with an ALA-enriched diet (flaxseeds, apples and carrots; FS) as previously described (20). Hamsters, aged 150 days, were anesthetized with urethane $(400 \mathrm{mg} / \mathrm{kg}$ i.p.) and sacrificed. Ventricles were rapidly excised, washed in cold PBS 1X, pH 7.4, weighed, frozen in liquid nitrogen, and stored at $-80^{\circ} \mathrm{C}$ until use. Alternatively, ventricles were processed for light or electron microscopy as described below. All experimental procedures were conducted in accordance with the 'Guide for Care and Use of Laboratory Animals' of the National Institutes of Health and were approved by the Institutional Animal Care and Use Committee of the University of Rome 'Tor Vergata'.

Western blot analysis. The electrophoresis of myocardium homogenates (100 $\mu \mathrm{g}$ protein/lane) from individual hamster heart ( $\mathrm{n}=5$ for each experimental group) was carried out in denaturing NuPAGE 4-12\% Bis-Tris-gels (Invitrogen, Italy), as previously described (22). Western blot filters were incubated with polyclonal antibodies against $\mathrm{N}$-cadherin, $\alpha-, \beta-$, $\gamma$-catenin (Santa Cruz, CA, USA) and connexin 43 (SigmaAldrich, MI, USA) and then developed with a Supersignal West Pico chemiluminescence kit (Pierce, Rockford, IL, USA). Equal loading and transfer of proteins was verified by Ponceau red staining of the filters. The intensity of the specific bands was evaluated using the Pro-Image 1.62 software, after blot scanning with an HP scanjet 4890 scanner, and expressed as densitometric units (D.U.) after normalization to the house-keeping internal controls $(21,23)$.

Immunohistochemistry. Hearts $(\mathrm{n}=5$ for each experimental group) from 150-day old GSH/PT, CMPH/PT and CMPH/FS were excised and fixed overnight in $4 \%$-buffered formalin. Hearts $(n=2)$ from 20-day old CMPH/PT were also included in the analysis. After paraffin embedding, each sample was dissected into 5- $\mu \mathrm{m}$ sections. Myocardial sections were then analysed for the reactivity to the specific polyclonal antibodies $(1 \mu \mathrm{g} / \mathrm{ml})$. Antigen-antibody complexes were visualised by 3-amino-9-ethylcarbazole (AEC) reaction as previously described $(24,25)$, using hematoxylin for counterstaining.
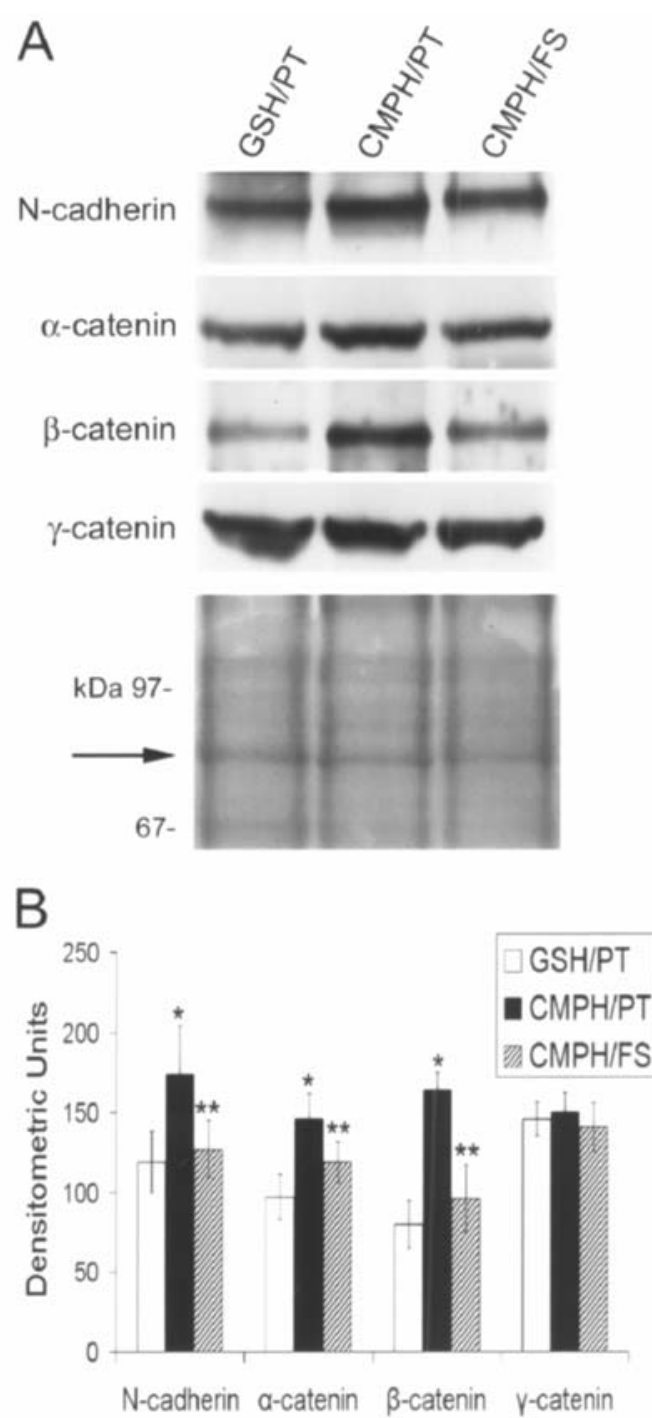

Figure 1. N-cadherin/catenins complex in ALA-fed cardiomyopatic hamster hearts. (A) Representative Western blot showing the reactivity of polyclonal antibodies to N-cadherin and catenins in the hearts of healthy and cardiomyopathic hamsters fed with a standard (GSH/PT and CMPH/PT) or an ALA-enriched diet (CMPH/FS). The arrow indicates a Ponceau red-stained protein band used as the housekeeping internal control. An additional internal control is represented by $\gamma$-catenin, whose expression levels were unaltered in the three experimental groups. (B) Densitometric analysis of Ncadherin and $\alpha^{-}, \beta_{-}$, and $\gamma$-catenin immunoblots were performed on five individual hearts per experimental group. The internal control band indicated in (A) was used for normalization of all densitometric values. ${ }^{*} \mathrm{CMPH} / \mathrm{PT}$ vs. GSH/PT: $\mathrm{p}=0.0098, \mathrm{p}=0.0012$ and $\mathrm{p}<0.0001$, for N-cadherin, $\alpha$-catenin and $\beta$-catenin, respectively. ${ }^{* *} \mathrm{CMPH} / \mathrm{FS}$ vs. CMPH/PT: $\mathrm{p}=0.019$, $\mathrm{p}=0.022$ and $\mathrm{p}=0.0002$, for $\mathrm{N}$-cadherin, $\alpha$-catenin and $\beta$-catenin, respectively. Bars indicate standard deviations.

Each observation was repeated twice. Staining intensity was semiquantitatively classified as negative $(-)$, weak $( \pm)$, moderate $(+)$, and high $(++/+++)$. No reactivity was observed when primary antibodies were replaced with normal rabbit serum.

Histopathology and ultrastructural analysis. Light microscopy and ultrastructural analyses were performed on myocardium fragments fixed in $2.5 \%$ glutaraldehyde in PBS pH 7.4. The samples were processed for transmission electron microscopy as previously described (26). For light microscopy, semithin sections were stained with tolouidine blue, according to routine 


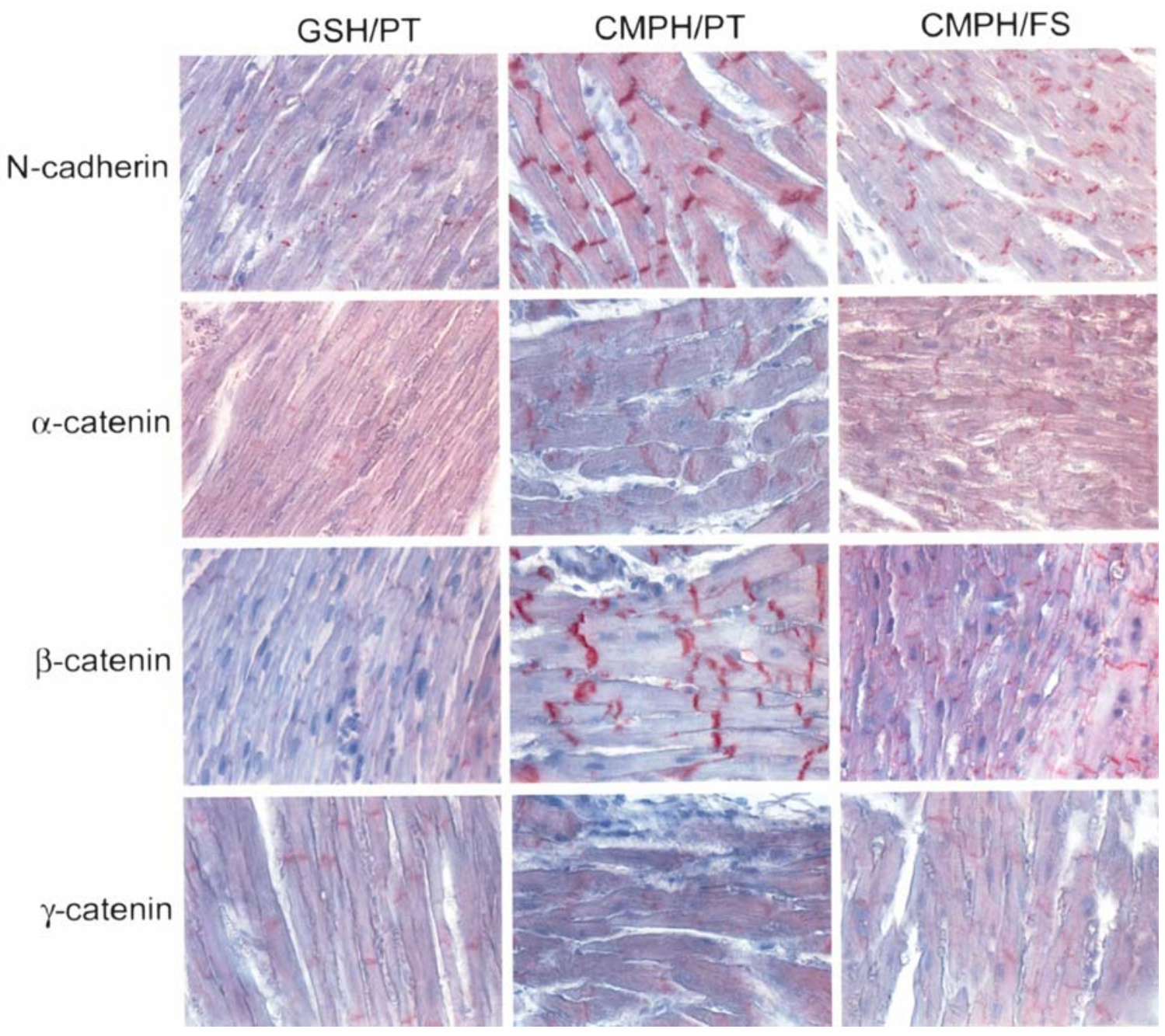

Figure 2. N-cadherin/catenin complex molecules in CMPH/FS vs. CMPH/PT cardiomyocyte intercalated disks. Representative immunohistochemical staining for $\mathrm{N}$-cadherin, and $\alpha^{-}, \beta-$, and $\gamma$-catenin in GSH/PT, CMPH/PT and CMPH/FS. Immunolabeling was counterstained with haematoxylin. Original magnification: $\mathrm{x} 400$.

procedures. Each observation was repeated twice using samples from three animals per each experimental group.

Statistical analysis. Associations among categorical variables were evaluated using Fisher's exact test and were considered significant at $p$-values $\leq 0.05$. Band densitometric values were compared using Student's t-test.

\section{Results}

$N$-cadherin/catenin complex accumulation in the cardiomyocyte intercalated disks of CMPH/FS vs. CMPH/PT. The $\mathrm{N}$-cadherin and catenins expression levels in 150-day old CMPH/PT vs. CMPH/FS were assessed by Western blot analysis on myocardium homogenates from five individual hearts. Age-matched control hamsters (GSH/PT) were also included in the analysis. Fig. 1A shows a representative experiment of $\mathrm{N}$-cadherin, $\alpha-, \beta-$, and $\gamma$-catenin detection by Western blotting, while Fig. 1B displays the densitometric units value (D.U.) average obtained in immunoblot experiments. The results confirm that, in $\mathrm{CMPH} / \mathrm{PT}$ vs. GSH/PT hearts, a higher expression level of N-cadherin (174 vs. 119 D.U.; $p=0.0098$ ), $\alpha$-catenin (146 vs. 97 D.U.; $p=0.0012$ ) and B-catenin (164 vs. 80 D.U.; p<0.0001) is detectable, whereas $\gamma$-catenin expression is unmodified. A substantial reduction from 174 to 127 D.U. ( $p=0.019), 146$ to 119 D.U. $(\mathrm{p}=0.022)$ and 164 to 96 D.U. $(\mathrm{p}=0.0002)$, respectively, affected the increased expression of $\mathrm{N}$-cadherin, $\alpha$ - and $\mathrm{B}$ catenin in CMPH/FS when compared with CMPH/PT hearts.

The expression of the $\mathrm{N}$-cadherin/catenin complex was further evaluated by immunohistochemical analysis. A representative experiment of $\mathrm{N}$-cadherin, $\alpha-, \beta$-, and $\gamma$-catenin immunostaining is shown in Fig. 2, while the results from repeated observations are summarized in Table I. Consistently with the immunoblot analysis, immunohistochemical results demonstrated that $\mathrm{N}$-cadherin/catenin complex molecules are differentially expressed in cardiomyocyte intercalated disks of CMPH/PT vs. CMPH/FS. In particular, the $\mathrm{N}$-cadherin expression was significantly decreased in the intercalated disks of 150-day old CMPH/FS ( $\mathrm{p}=0.0079$ ), as compared to the augmented expression in the intercalated disks of CMPH/PT hearts. Similarly, both $\alpha$ - and $\beta$-catenin, accumulated along with Ncadherin in the intercalated disks of CMPH/PT as compared to GSH/PT, showed reduced expression levels in CMPH/FS: the $\alpha$-catenin expression decreased to levels similar to those 
Table I. Immunohistochemical expression of N-cadherin/catenins complex molecules and connexin 43 in GSH/PT, CMPH/PT and $\mathrm{CMPH} / \mathrm{FS}$ intercalated disks.

\begin{tabular}{|c|c|c|c|c|c|c|c|c|c|}
\hline & \multicolumn{3}{|c|}{ GSH/PT (n. 5) } & \multicolumn{3}{|c|}{ CMPH/PT (n. 5) } & \multicolumn{3}{|c|}{ CMPH/FS (n. 5) } \\
\hline & \multicolumn{3}{|c|}{$\begin{array}{l}\text { Intercalated disks } \\
\text { staining intensity }\end{array}$} & \multicolumn{3}{|c|}{$\begin{array}{l}\text { Intercalated disks } \\
\text { staining intensity }\end{array}$} & \multicolumn{3}{|c|}{$\begin{array}{l}\text { Intercalated disks } \\
\text { staining intensity }\end{array}$} \\
\hline & \pm & + & $++/+++$ & \pm & + & $++/+++$ & \pm & + & $++/+++$ \\
\hline $\mathrm{N}$-cadherin & 5 & 0 & 0 & 0 & 0 & 5 & 0 & 5 & 0 \\
\hline$\alpha$-catenin & 5 & 0 & 0 & 0 & 5 & 0 & $5^{\mathrm{b}}$ & 0 & 0 \\
\hline ß-catenin & 5 & 0 & 0 & 0 & 0 & 5 & 0 & 5 & 0 \\
\hline$\gamma$-catenin & 5 & 0 & 0 & 5 & 0 & 0 & 5 & 0 & 0 \\
\hline \multirow[t]{2}{*}{ Connexin 43} & 0 & 0 & 5 & 0 & 5 & 0 & 0 & 5 & 0 \\
\hline & & $A^{d}$ & $\mathrm{~B}^{\mathrm{e}}$ & & A & B & & A & $\mathrm{B}$ \\
\hline Connexin 43 & & 15 & $0 / 5$ & & $5 / 5$ & $0 / 5$ & & $0 / 5$ & $5 / 5^{\mathrm{f}}$ \\
\hline
\end{tabular}

${ }_{\mathrm{a}}^{\mathrm{a}}=0.0079 \mathrm{CMPH} / \mathrm{FS}$ vs. CMPH/PT, Fisher's exact test; ${ }^{\mathrm{b}} \mathrm{p}=0.0079 \mathrm{CMPH} / \mathrm{FS}$ vs. CMPH/PT, Fisher's exact test; ${ }^{\mathrm{c}} \mathrm{p}=0.0079 \mathrm{CMPH} / \mathrm{FS}$ vs. $\mathrm{CMPH} / \mathrm{PT}$, Fisher's exact test. ${ }^{\mathrm{d}}$ Localization restricted to intercalated disks. ${ }^{\mathrm{e}}$ Intercalated disks plus lateral cell borders localization.

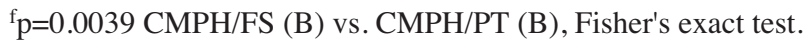

A
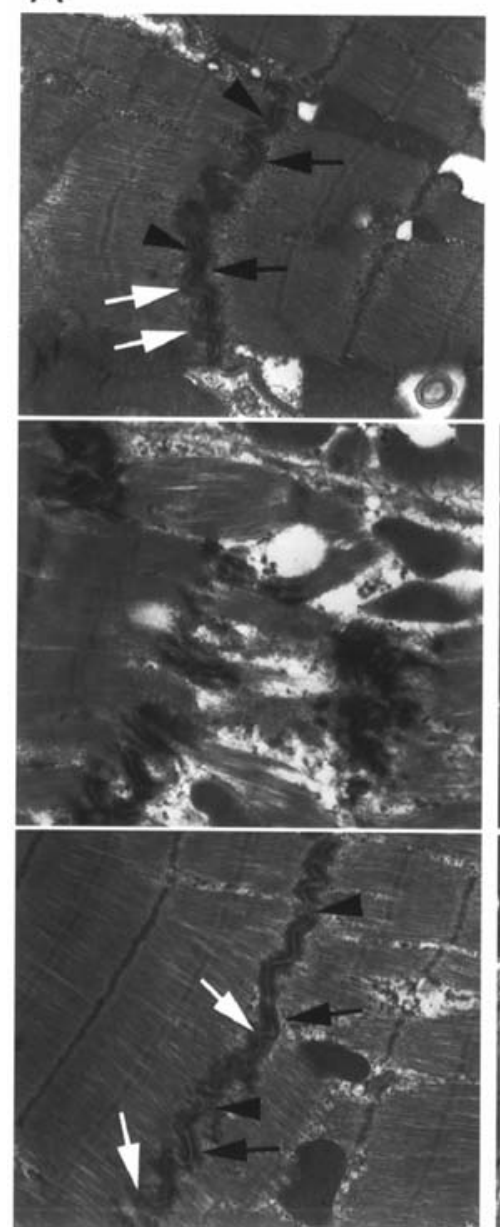

B

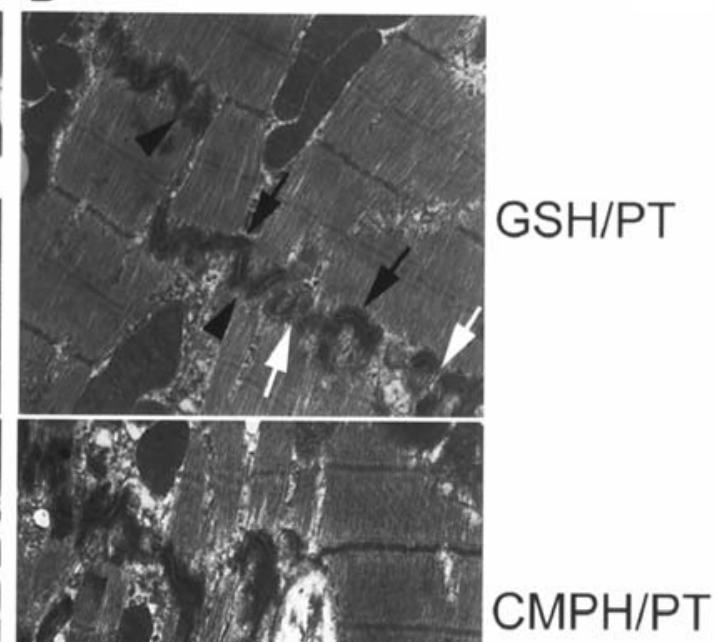

$\mathrm{CMPH} / \mathrm{PT}$

Figure 3. Intercalated disk remodeling in hamsters fed with ALA-enriched diet. Intercalated disk ultrastructure (lengthwise cut) in two representative hearts (A and B) from GSH/PT, CMPH/PT and CMPH/FS. In intercalated disks of healthy GSH/PT it is possible to distinguish the periodicity of adherens junctions (white arrows), desmosomes (black arrows) and gap junctions (arrowheads). This ordered organization is lost in the intercalated disks of CMPH/PT, which appear chaotically located and structurally swirling, but is restored in the hearts from CMPH/FS, in which the periodicity of adherens junctions (white arrows), desmosomes (black arrows) and gap junctions (arrowheads), is again distinguishable. Original magnification: x15500. 
A

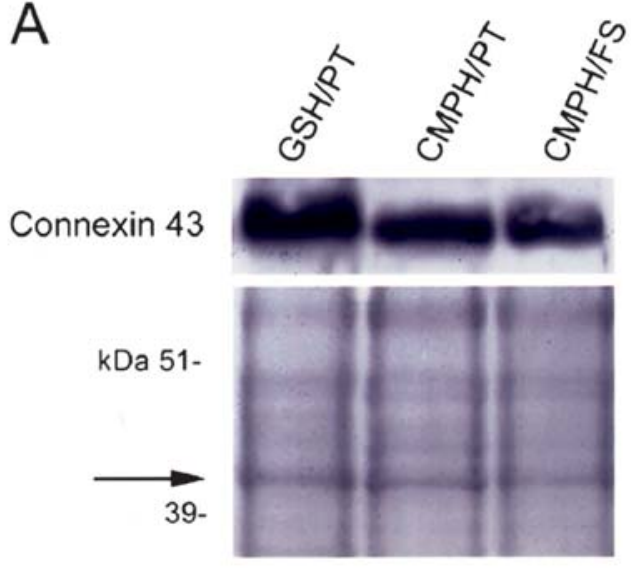

B

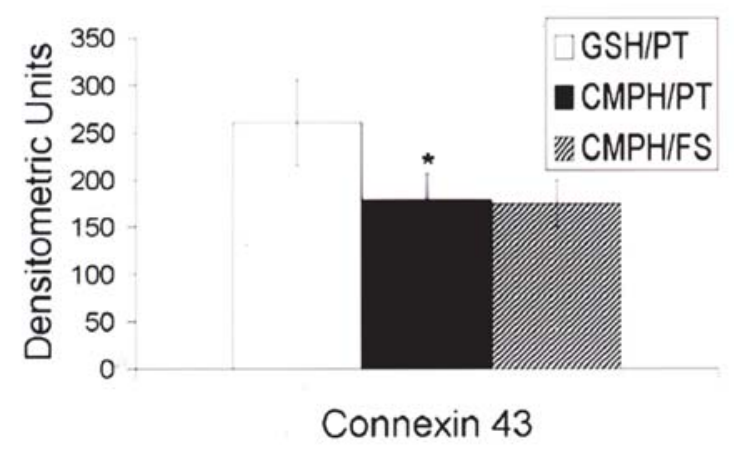

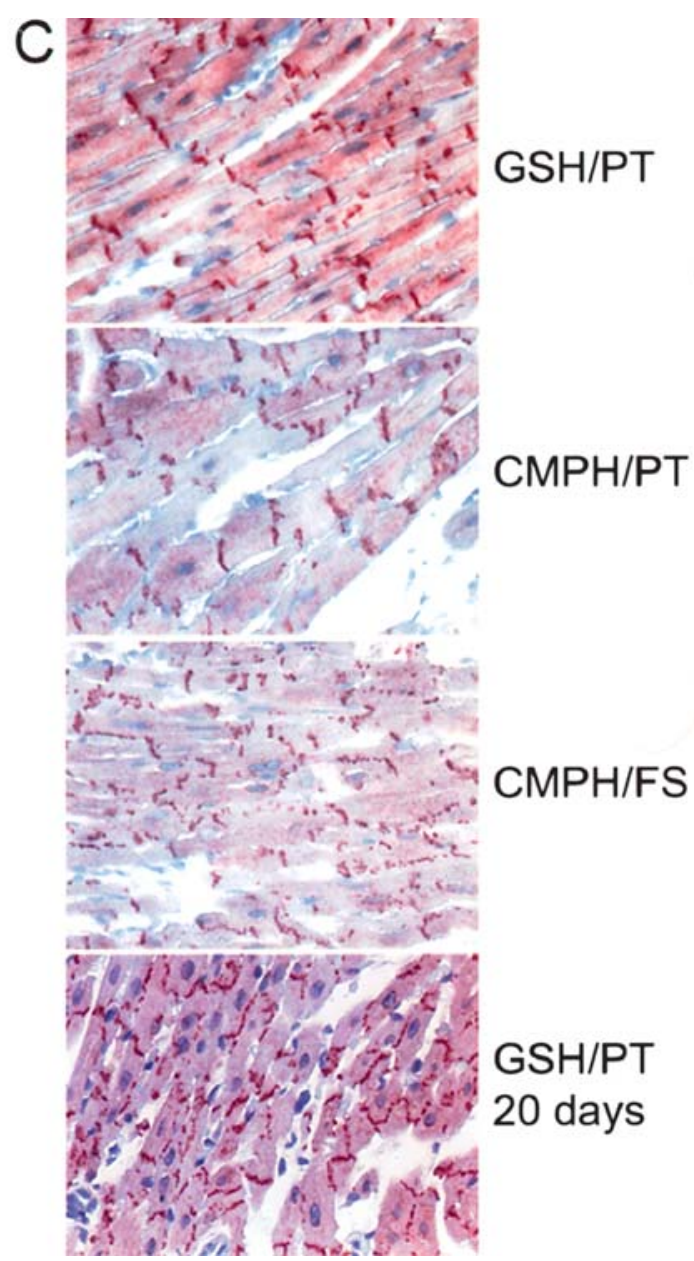

Figure 4. The ALA-enriched diet affects connexin 43 localization, but not its expression in CMPH hearts. (A) Representative Western blot showing a decreased connexin 43 signal in CMPH/PT and CMPH/FS, as compared to GSH/PT. The arrow indicates a Ponceau red-stained protein band used as the housekeeping internal control. (B) Densitometric analysis of connexin 43 immunoblots performed on five individual hearts per experimental group. The internal control band indicated in (A) was used for normalization of densitometric values. ${ }^{*} \mathrm{CMPH} / \mathrm{PT}$ vs. GSH/PT: $\mathrm{p}=0.01$. Bars indicate standard deviations. (C) Representative immunolocalization of connexin 43 in intercalated disks of 150-day old GSH/PT and CMPH/PT, and in both intercalated disks and cardiomyocyte lateral cell borders in age-matched CMPH/FS; connexin 43 distribution in 150 day-old CMPH/FS resembled that found in immature cardiomyocytes of 20-day old healthy GSH/PT. Immunolabeling was counterstained with haematoxylin. Original magnification: x400.

found in GSH/PT ( $\mathrm{p}=0.0079)$, while $\beta$-catenin reduced its expression from high to moderate $(\mathrm{p}=0.0079) . \gamma$-catenin immunostaining was similar in 150-day old CMPH/PT and $\mathrm{CMPH} / \mathrm{FS}$, thus corroborating the results obtained by Western blot analysis.

The impairment of $C M P H$ intercalated disk ultrastructure is counteracted by the ALA-enriched diet. Representative pictures of GSH/PT, CMPH/PT and CMPH/FS intercalated disks by transmission electron microscopy are shown in Fig. 3. In CMPH/PT, the intercalated disks are chaotically located, structurally redundant, swirling, and lacking the typical periodicity of their structural components including desmosomes, adherens and gap junctions. Conversely, intercalated disks of CMPH/FS closely resemble the structure of those found in GSH/PT healthy hearts. CMPH/FS intercalated disks are characterized by an ondulating double membrane separating two adiacent cardiomyocytes and the three types of specialised junctions, including desmosomes, adherens and gap junctions, are readily recognizable. On the whole, the
ALA-enriched diet demonstrates its effects in counteracting the pathological alterations typically found in $\mathrm{CMPH}$ intercalated disks at the ultrastructural level, as well as at the molecular level.

Delocalization of connexin 43 on the lateral plasmalemma by the ALA-enriched diet. Among the different connexins, at least four (CX37, CX40, CX43 and CX45) are found in the heart. Within this group, CX43 is the major isoform expressed in cardiomyocytes. The n-3 polyunsaturated fatty acids are able to modulate the astrocyte membrane fatty acid composition and affect gap junction function (27). In order to determine the potential effects of the ALA-enriched diet on the CX43 expression level and distribution in the hearts of CMPH/PT vs. CMPH/FS, myocardium homogenates and tissue sections were subjected to Western blot and immunohistochemical analysis, respectively. Hearts from GSH/PT were included in these experiments as controls. Fig. 4A shows a representative Western blot, in which the myocardial expression of CX43 was significantly reduced in $\mathrm{CMPH} / \mathrm{PT}$, as compared to 


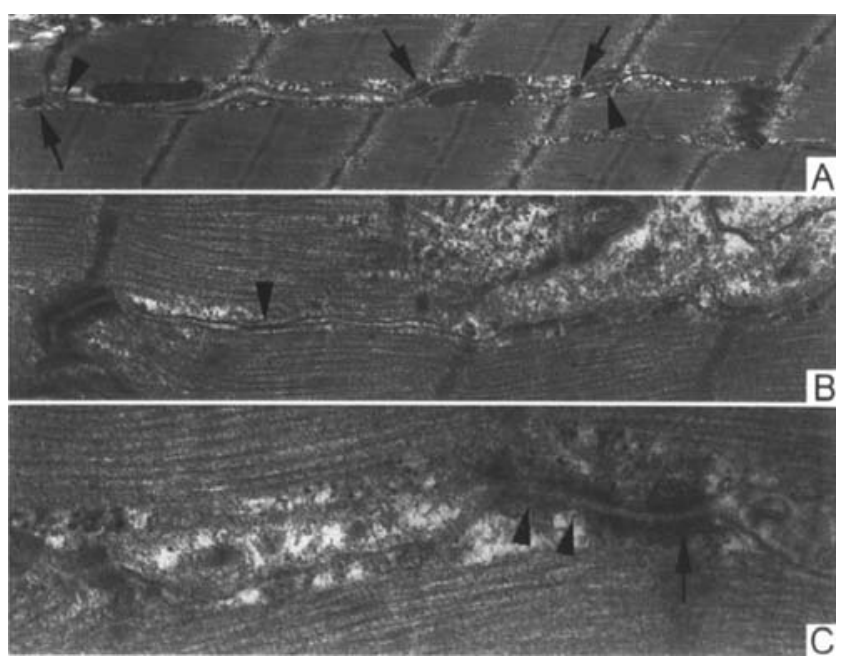

Figure 5. Junctional complexes at the lateral border of CMPH/FS cardiomyocytes. Presence of junctional complexes including gap junctions (arrowheads) and desmosomes (arrows) outside the intercalated disks, at the lateral CMPH/FS cardiomyocyte border (lengthwise cut). Transmission electron microscopy. Original magnification: A: x21000, B: x28500, C: x 52000 .

healthy GSH/PT (260 vs. 179 D.U.; p=0.01) (Fig. 4B). The ALA-enriched diet was not apparently able to modify CX43 myocardial levels in CMPH/FS vs. CMPH/PT hearts (Fig. 4A and $\mathrm{B}$ ). Nonetheless, the ALA-enriched diet induced a redistribution of CX43, as determined by immunohistochemical analysis (Fig. 4C). While in both 150-day old GSH/PT and CMPH/PT the CX43 expression was exclusively present in the intercalated disks, in age-matched CMPH/FS it was relocated in the lateral cell borders $(p=0.0039)$ (Fig. 4C and Table I). It is worth noting that the presence of CX43 at the lateral border of cardiomyocytes in 150 day-old CMPH/FS resembled the distribution observed in immature cardiomyocytes of 20 day-old healthy GSH/PT (Fig. 4C). Since CX43 is the major component of gap junctions, the transmission electron microscopy was used to evaluate the possible presence of gap junctions between the lateral borders of two adjacent cardiomyocytes. Fig. 5 shows a representative peculiar feature of the CMPH/FS cardiomyocytes in which, at high magnification, it is possible to discern the presence of junctional complexes, including gap junctions and desmosomes, located outside the intercalated disks at the lateral cell border. These ultrastructural findings, corroborate the immunohistochemical distribution of CX43 observed in the CMPH/FS myocardium.

\section{Discussion}

Marine- and plant-derived polyunsaturated n-3 fatty acid (PUFA) dietary consumption is beneficial for patients affected by cardiovascular diseases (28-36). However, the mechanisms through which these fatty acids exert their effects on the cardiovascular system remain to be elucidated. Anti-arrythmic as well as anti-inflammatory and antithrombogenic mechanisms have been proposed, but none exhaustively explains PUFA effects. It has been demonstrated that PUFAs show an overall plasmalemma protection in hereditary cardiomyopathic animals with widespread cardiomyocyte damage. As a consequence of the PUFA administration, most of the transmembrane proteins preserved their physiological expression and location and the intracellular signalling and gene expression were maintained within the normal range. The ultimate consequence was that the diet-treated-animals were no longer affected by heart failure and their longevity was extraordinarily extended: when all cardiomyopathic animals were deceased, $>60 \%$ of the PUFA diet-treated animals were still alive and apparently healthy (20). Conceivably, all previously reported effects, such as the anti-arrhythmic and anti-inflammatory effects, should be considered as consequence of the regularization of the cell signalling induced by PUFAs. Therefore, the elucidation of the PUFA's modulatory capability in respect to the cardiomyocyte intracellular signalling is prerequisite to assess the actual cardioprotective potential and to envisage a controlled and efficient use in clinical practice.

Among the most important intracellular signalling cascades is the cadherin/catenin complex (CCC). CCC has a dual role: it provides nuclei with some of the most important signals from the external side of the membrane, and acts as a fundamental adhesion mechanism among cells. In particular, the $\beta$-catenin interacts with cadherin in the adhesion processes and, interrelating with the Wnt/Wingless signalling cascade, associates with the LEF/TCF transcription factor in the nucleus (37). In a previous study, an abnormal accumulation of the $\mathrm{N}$-cadherin/catenin complex was detected in the cardiomyocyte intercalated disks of CMPH/PT (21).

The present investigation demonstrates that this abnormal CCC accumulation can be prevented by feeding CMPHs with a diet enriched with ALA and, thus, changing the plasmalemmal lipid composition (20). Western blotting and immunohistochemistry showed that the expression of N-cadherin, $\alpha$ - and $\beta$-catenin in cardiomyocyte intercalated disks of $\mathrm{CMPH} / \mathrm{FS}$ is as low as that found in healthy hamster (GSH/PT) hearts. In addition to the normalized CCC expression, the overall ultrastructure of the cardiomyocyte intercalated disks is re-established in CMPH fed the ALA-enriched diet. Collectively, these data along with previously reported observations (20), strongly suggest that, modifying the plasmalemmal lipid composition by increasing the relative presence of ALA and of self-synthesized EPA (20), cardiomyocytes display an augmented capability to counteract major events perturbing its physical and functional integrity, such as the deletion of the $\delta$-sarcoglycan, a protein contributing to stabilize cell/cell contacts $(17,18)$. As a consequence, an improvement of major intracellular signalling cascades may occur and counteract the appearance of the cell, tissue and organ pathological features. However, ALA is not able to reconstitute ad integrum the cell structure and function. Consistently, in CMPH/FS, the CX43 myocardial expression, that is substantially reduced in $\mathrm{CMPH} / \mathrm{PT}$ as compared to healthy GSH/PT, is not restored to normal levels. In addition, the CX43 expression is peculiarly delocalized on the lateral plasmamembrane of cardiomyocytes, while gap junctions are present between the lateral borders of adjacent cardiomyocytes of ALA-fed CMPHs.

Although CX43 is not required for the organization of intercalated disk adhesive junctions in adult cardiomyocytes 
(4), the derangement of the gap junction organization and/or reduced CX43 expression are associated with an increased arrhythmic tendency in heart disease (10), the perturbation of the cardiomyocyte electrical coupling and a decreased ventricular conduction velocity (39). Furthermore, alterations of connexin expression and/or subcellular distribution occur in different potentially arrhythmogenic cardiac diseases $(10,11,40)$. Conversely, the CX43 upregulation and lateralization are important events of the immediate adaptive response of the heart to the increased workload in compensated hypertrophy (38). Nevertheless, the actual relevance of the gap junctional complexes located at the lateral CMPH cardiomyocyte borders remains to be understood. However, it can be speculated that, as the gap junctions are essential for the coordinated contraction of the heart (38) and CX43 is the main connexin isoform involved in the building of myocardial gap junctions $(11,38)$, the lateral localization of the proteins and the related cell structures detected in the CMPH/FS hearts could represent a strategy that the damaged cardiomyocytes apply to facilitate the propagation of the electric stimulus and the contractile cell recruitment through the myocardium in order to overwhelm the possible local depolarization that can be self-generated by the perturbed plasmalemma and the consequent arrhythmogenic potential of the myocardial disease.

On the whole, this study demonstrates a substantial effect of an ALA-enriched diet on cardiomyocyte intercalated disk structure and molecular composition and further supports the possible role of $\omega-3$ polyunsatured fatty acids in the prevention of cardiac disease.

\section{Acknowledgements}

This study was supported by grants from PRIN 2005 and AIRC Regionale (A. Modesti), PRIN 2004 (L. Masuelli). We are grateful to Barbara Bulgarini for her editing assistance.

\section{References}

1. Forbes MS and Sperelakis N: Intercalated discs of mammalian heart: a review of structure and function. Tissue Cell 17: 605-648, 1985.

2. Severs NJ: Intercellular junctions and the cardiac intercalated disk. Adv Myocardiol 5: 223-242, 1985.

3. Zuppinger C, Eppenberger-Eberhardt $\mathrm{M}$ and Eppenberger HM: $\mathrm{N}$-cadherin: structure, function and importance in the formation of new intercalated disc-like cell contacts in cardiomyocytes. Heart Fail Rev 5: 251-257, 2000.

4. Gutstein DE, Liu FY, Meyers MB, Choo A and Fishman GI: The organization of adherens junctions and desmosomes at the cardiac intercalated disc is independent of gap junctions. J Cell Sci 116: 875-885, 2003.

5. van Gijn ME, Daemen MJ, Smits JF and Blankesteijn WM: The wnt-frizzled cascade in cardiovascular disease. Cardiovasc Res 55: 16-24, 2002.

6. Peralta Soler A and Knudsen KA: N-cadherin involvement in cardiac myocyte interaction and myofibrillogenesis. Dev Biol 162: 9-17, 1994.

7. Linask KK, Knudsen KA and Gui YH: N-cadherin-catenin interaction: necessary component of cardiac cell compartmentalization during early vertebrate heart development. Dev Biol 185: 148-164, 1997.

8. Takeichi M: The cadherins: cell-cell adhesion molecules controlling animal morphogenesis. Development 102: 639-655, 1988.

9. Ahmad F, Seidman JG and Seidman CE: The genetic basis for cardiac remodeling. Annu Rev Genomics Hum Genet 6: 185-216, 2005 .
10. Severs NJ, Coppen SR, Dupont E, Yeh HI, Ko YS and Matsushita T: Gap junction alterations in human cardiac disease. Cardiovasc Res 62: 368-377, 2004.

11. Dupont E, Matsushita T, Kaba RA, et al: Altered connexin expression in human congestive heart failure. J Mol Cell Cardiol 33: 359-371, 2001.

12. Borrmann CM, Grund C, Kuhn C, Hofmann I, Pieperhoff S and Franke WW: The area composita of adhering junctions connecting heart muscle cells of vertebrates. II. Colocalizations of desmosomal and fascia adhaerens molecules in the intercalated disk. Eur J Cell Biol 85: 469-485, 2006.

13. Sheikh F, Chen Y, Liang X, et al: alpha-E-catenin inactivation disrupts the cardiomyocyte adherens junction, resulting in cardiomyopathy and susceptibility to wall rupture. Circulation 114: 1046-1055, 2006.

14. Ehler E and Perriard JC: Cardiomyocyte cytoskeleton and myofibrillogenesis in healthy and diseased heart. Heart Fail Rev 5: 259-269, 2000.

15. Ferreira-Cornwell MC, Luo Y, Narula N, Lenox JM, Lieberman $M$ and Radice GL: Remodeling the intercalated disc leads to cardiomyopathy in mice misexpressing cadherins in the heart. J Cell Sci 115: 1623-1634, 2002.

16. Nigro V, Okazaki Y, Belsito A, et al: Identification of the Syrian hamster cardiomyopathy gene. Hum Mol Genet 6: 601-607, 1997.

17. Sakamoto A, Ono K, Abe M, et al: Both hypertrophic and dilated cardiomyopathies are caused by mutation of the same gene, delta-sarcoglycan, in hamster: an animal model of disrupted dystrophin-associated glycoprotein complex. Proc Natl Acad Sci USA 94: 13873-13888, 1997.

18. Straub V, Duclos F, Venzke DP, Lee JC, Cutshall S, Leveille CJ and Campbell KP: Molecular pathogenesis of muscle degeneration in the delta-sarcoglycan-deficient hamster. Am J Pathol 153: 1623-1630, 1998.

19. Lipskaia L, Pinet C, Fromes Y, Hatem S, Cantaloube I, Coulombe A and Lompré AM: Mutation of $\delta$-sarcoglycan is associated with $\mathrm{Ca}^{+}$-dependent vascular remodeling in the Syrian Hamster. Am J Pathol 171: 162-171, 2007.

20. Fiaccavento R, Carotenuto F, Minieri M, et al: Alpha-linolenic acid-enriched diet prevents myocardial damage and expands longevity in cardiomyopathic hamsters. Am J Pathol 169: 1913-1924, 2006.

21. Masuelli L, Bei R, Sacchetti P, et al: Beta-catenin accumulates in intercalated disks of hypertrophic cardiomyopathic hearts. Cardiovasc Res 60: 376-387, 2003.

22. Bei R, Mentuccia D, Trono P, et al: Immunity to extracellular matrix antigens is associated with ultrastructural alterations of the stroma and stratified epithelium basement membrane in the skin of Hashimoto's thyroiditis patients. Int J Immunopathol Pharmacol 19: 661-674, 2006.

23. Bei R, Budillon A, Reale MG, et al: Cryptic epitopes on alphafetoprotein induce spontaneous immune responses in hepatocellular carcinoma, liver cirrhosis, and chronic hepatitis patients. Cancer Res 59: 5471-5474, 1999.

24. Bei R, Budillon A, Masuelli L, et al: Frequent overexpression of multiple ErbB receptors by head and neck squamous cell carcinoma contrasts with rare antibody immunity in patients. J Pathol 204: 317-325, 2004.

25. Bei R, Moretti A, Visco V, De Filippi R, Tsang KY, Frati L and Muraro R: Cell mediated cytotoxicity of human colon carcinoma cells by a monoclonal antibody (R4) recognizing the carcinoembryonic antigen (CEA) and CEA related-molecules. Int J Oncol 8: 1127-1135, 1996.

26. Masuelli L, Focaccetti C, Cereda V, et al: Gene-specific inhibition of breast carcinoma in BALB-neuT mice by active immunization with rat $\mathrm{Neu}$ or human ErbB receptors. Int $\mathrm{J}$ Oncol 30: 381-392, 2007.

27. Champeil-Potokar G, Chaumontet C, Guesnet P, Lavialle M and Denis I: Docosahexaenoic acid (22:6n-3) enrichment of membrane phospholipids increases gap junction coupling capacity in cultured astrocytes. Eur J Neurosci 24: 3084-3090, 2006.

28. Simopoulos AP: Omega-3 fatty acids in the preventionmanagement of cardiovascular disease. Can J Physiol Pharmacol 75: 234-239, 1997.

29. Holub DJ and Holub BJ: Omega-3 fatty acids from fish oils and cardiovascular disease. Mol Cell Biochem 263: 217-225, 2004.

30. Breslow JL: n-3 fatty acids and cardiovascular disease. Am J Clin Nutr 83 (Suppl 6): 1477-1482, 2006. 
31. Psota TL, Gebauer SK and Kris-Etherton P: Dietary omega-3 fatty acid intake and cardiovascular risk. Am J Cardiol 98: 3-18, 2006.

32. Angerer $P$ and von Schacky C: n-3 polyunsaturated fatty acids and the cardiovascular system. Curr Opin Lipidol 11: 57-63, 2000.

33. Kris-Etherton PM, Harris WS and Appel LJ; American Heart Association, Nutrition Committee: Fish consumption, fish oil, omega-3 fatty acids, and cardiovascular disease. Circulation 106: 2747-2757, 2002.

34. Harper CR and Jacobson TA: Usefulness of omega-3 fatty acids and the prevention of coronary heart disease. Am J Cardiol 96: 1521-1529, 2005.

35. Calder PC: n-3 fatty acids and cardiovascular disease: evidence explained and mechanisms explored. Clin Sci 107: 1-11, 2004.

36. Mantzioris E, Cleland LG, Gibson RA, Neumann MA, Demasi M and James MJ: Biochemical effects of a diet containing foods enriched with n-3 fatty acids. Am J Clin Nutr 72: 42-48, 2000.
37. Miller JR, Hocking AM, Brown JD and Moon RT: Mechanism and function of signal transduction by the Wnt/beta-catenin and Wnt/Ca2 ${ }^{+}$pathways. Oncogene 18: 7860-7872, 1999.

38. Kostin S, Dammer S, Hein S, Klovekorn WP, Bauer EP and Schaper J: Connexin 43 expression and distribution in compensated and decompensated cardiac hypertrophy in patients with aortic stenosis. Cardiovasc Res 62: 426-436, 2004.

39. Li J, Patel VV, Kostetskii I, et al: Cardiac-specific loss of $\mathrm{N}$-cadherin leads to alteration in connexins with conduction slowing and arrhythmogenesis. Circ Res 97: 474-481, 2005.

40. Peters NS, Coromilas J, Severs NJ and Wit AL: Disturbed connexin43 gap junction distribution correlates with the location of reentrant circuits in the epicardial border zone of healing canine infarcts that cause ventricular tachycardia. Circulation 95: 988-996, 1997. 